\title{
The new UK antimicrobial resistance strategy and action plan
}

\author{
A major societal, political, clinical, and research challenge
}

\author{
Anthony S Kessel honorary professor ${ }^{1}$, Mike Sharland professor in paediatric infectious diseases ${ }^{2}$ \\ ${ }^{1}$ Faculty of Public Health and Policy, London School of Hygiene and Tropical Medicine, London, UK; ${ }^{2}$ Paediatric Infectious Diseases Research \\ Group, St George's University London, London SW17 ORE, UK
}

This week the chief medical officer highlighted in her report how the rise of antimicrobial resistance (AMR) poses a threat to healthcare delivery in the United Kingdom. ${ }^{1}$ This will be followed shortly by the Department of Health's new UK Five Year Antimicrobial Resistance Strategy and Action Plan, which will reflect the need for a clear change in the understanding and response to AMR by the public, the NHS, and the government in the UK. The rise of AMR as a serious health threat is due to the international spread of multidrug resistant (MDR) Gram negative bacteria, the global overuse of antibiotics in humans and animals, and the almost complete lack of new antibiotic development. ${ }^{2}$ All of these are now of direct concern to the NHS.

The $85 \%$ reduction in rates of meticillin resistant Staphylococcus aureus (MRSA) bloodstream infections seen in England between 2003 and 2011 has been remarkable. MRSA is now responsible for less than 2\% of all bloodstream infections in England. Less remarked on has been the inexorable rise in the number of bloodstream infections attributable to Gram negative organisms (particularly Escherichia coli), which now comprise more than half of the around 100000 of these infections reported in England annually. ${ }^{3}$ Most large NHS hospitals now identify 50-100 times more patients with Gram negative bloodstream infections than those with MRSA, with antibiotic resistance rates of $10-20 \%$ and mortality rates of $30 \%$ reported for MDR forms. ${ }^{4}$ In England the successful introduction of conjugate pneumococcal vaccine means that the number of reported Klebsiella pneumoniae bloodstream infections in England is now higher than for Streptococcus pneumoniae.

In many European countries AMR rates are much worse. In 2011 the European Centre for Disease Prevention and Control reported a significant increase in multidrug resistant $E$ coli and $K$ pneumoniae (for example, resistance to third generation cephalosporins, fluoroquinolones, and aminoglycosides) in more than a third of European Union/European Economic Area countries. ${ }^{5}$ Klebsiella is an important pathogen in the spread of resistance. Many antibiotic resistance genes group together in plasmids easily transferred between bacteria, with particular clones carrying multiple resistance genes (for example, OXA-48 and CTX-M15). Many EU countries are now reporting Klebsiella MDR rates of 25-40\%.

Globally, rates of MDR Gram negative bacterial infection can be even higher. ${ }^{6}$ This has inevitably led to a rapid rise in the use of carbapenem antibiotics (for example, meropenem) as empirical treatment for suspected sepsis. In turn, this has led to a rapid increase in hospital outbreaks of carbapenemase producing organisms, which are usually sensitive to only one or two older less effective antibiotics. In the UK, there has also been a sharp rise in meropenem use and increasing reports of carbapenemase producing organisms, with worryingly both clonal outbreaks and interspecies plasmid spread seen in some NHS hospitals. Only one or two new antibiotics that target Gram negative organisms are likely to be marketed in the next decade (http://antibiotic-action.com), which raises the concern that virtually untreatable infections will threaten routine NHS care for vulnerable children and adults. Experts in policy analysis describe this type of problem as a "super wicked challenge."

The new UK strategy is an important step in recognising and responding to these concerns, driven by the emergence of carbapenemase producing organisms in the NHS. At its core the strategy recognises that AMR, infection prevention and control, and antimicrobial stewardship are closely interconnected and all need to be strengthened. The seven aims (table $\downarrow$ ) reflect that all individuals and organisations have unique roles and responsibilities. Enhanced infection prevention and control are crucial to limiting the spread of MDR Gram negative bacteria, both into and across the NHS.

New challenges will include screening (by rectal swab) and isolation of any patient admitted to the NHS who has received inpatient care outside the UK, with rigorous control of any outbreaks of multidrug resistant infection inside the NHS. Acute trusts and their boards will need to learn a new language and consider how to strengthen infection prevention and control practice using new methods of organisational and behavioural change to reinforce policy implementation. Although zero tolerance to MRSA and Clostridium difficile infection remain 
crucial, these organisms now represent only a small part of healthcare associated infections. Enterobacteraciae (Gram negative organisms) were the most common cause identified in the recent national healthcare associated infections point prevalence survey (32.4\% of reported organisms $v 2.4 \%$ for MRSA). ${ }^{3}$

Antimicrobial prescribing needs to be more evidence based and more efficiently targeted. New NHS initiatives to provide antimicrobial stewardship guidance in secondary care (Start Smart then Focus) and primary care (TARGET antimicrobial toolkit) ${ }^{8}$ need to develop into more formal quality indicators. They must emphasise that every prescriber should consider the impact of each prescription on the individual patient and the wider burden of AMR. The risk-benefit balance for antibiotic prescribing is becoming even more complex; patient expectations, clinical outcomes, and ethical issues must all be considered.

This strategy makes the UK the first country to explicitly announce its intention to develop national outcome measures in AMR using specific drug-bug combination resistance rates (for example, rates of $E$ coli resistance to third generation cephalosporins). This is a brave move and should be welcomed. The chief medical officer has taken a clear leadership role by tackling the international dimensions of the problem, adding AMR to the Department of Health risk register and calling for AMR to be added to the national risk register (National Security Risk Assessment) to promote cross government action.

Important areas that will be covered include antimicrobial use in animals and new initiatives to encourage the development of novel antimicrobials. ${ }^{1}$

The wider application of molecular microbiology, particularly whole genome sequencing, to detect clonal spread of MDR Gram negative bacteria within hospitals is providing a rapid explosion of new data. It is still unclear if this will lead to effective new control policies. The research agenda is extensive, but the NHS information technology and National Institute of Health Research infrastructures are well placed to provide global leadership in this area. ${ }^{9}$ New technology focusing on rapid diagnosis of specific bacteria and resistance genes, along with combination biomarkers indicating bacterial or viral infections, especially if adapted to near patient testing, could have a major impact on targeting appropriate antibiotic treatment. Improved surveillance by Public Health England, using large dataset linkage combined efficiently with observational studies focused on clinical outcomes, including all infection related deaths, will also help to define new targets for intervention.
The UK strategy provides a call for action. The problems are global and the terminology complex, but the importance is clear. Public Health England and the NHS Commissioning Board will play major roles in implementing the strategy. A fundamental standard of the NHS should include basic high quality routine infection control and clinical care, as noted by the Francis inquiry. ${ }^{10}$ These standards of care are crucial to the prevention and control of all healthcare associated infections, including MDR Gram negative bacteria.

Competing interests: 1/we have read and understood the BMJ Group policy on declaration of interests and declare the following interests: AK is director of public health strategy and medical director at the Health Protection Agency, where he chairs the agency's programme board on healthcare associated infections and antimicrobial resistance and stewardship; he will be transferring to Public Health England (PHE) on 1 April 2013. AK had an opportunity to comment on the strategy in draft form. The views expressed here are personal and do not represent those of the agency or of PHE. MS is chair of the Department of Health Expert Advisory Committee on Antimicrobial Resistance and Healthcare Associated Infection (ARHAl). The committee had the opportunity to comment on the strategy in draft form. The views expressed here are personal and do not represent those of ARHAI.

Provenance and peer review: Not commissioned; externally peer reviewed.

1. Department of Health. Annual report of the chief medical officer. Vol 2 Infections and the rise of antimicrobial resistance. 2011. https://www.wp.dh.gov.uk/publications/files/2013/ rise of antimicrobial resistance. 2011. https://ww

2 Wise R. The urgent need for new antibacterial agents $J$ Antimicrob Chemother 2011;66:1939-40.

3 Health protection Agency. Healthcare associated infections (HCAl). www.hpa.org.uk/ Topics/InfectiousDiseases/InfectionsAZ/HCAl/.

4 De Kraker ME, Davey PG, Grundmann H; BURDEN study group. Mortality and hospital stay associated with resistant Staphylococcus aureus and Escherichia coli bacteremia: estimating the burden of antibiotic resistance in Europe. PLOS Med 2011;8:e1001104.

5 European Centre for Disease Prevention and Control. Antimicrobial resistance surveillance in Europe 2011. 2012. http://ecdc.europa.eu/en/publications/Publications/Forms/ECDC_ DispForm. aspx?ID=998.

6 WHO. The evolving threat of antimicrobial resistance-options for action. 2012. www. who.int/patientsafety/implementation/amr/publication/en/index.html.

7 Levin K, Cashore B, Bernstein S, Auld G. Overcoming the tragedy of super wicked problems: constraining our future selves to ameliorate global climate change. Policy Sci 2012;45:123-52.

8 Ashiru-Oredope D, Sharland M, Charani E, McNulty C, Cooke J; ARHAI Antimicrobial Stewardship Group. Improving the quality of antibiotic prescribing in the NHS by developing a new Antimicrobial Stewardship Programme: Start Smart then Focus. J Antimicrob Chemother 2012;67(suppl 1):i51-63.

9 HM Government. PM Speech on life sciences and opening up the NHS. 2011. www. number10.gov.uk/news/pm-speech-on-life-sciences-and-opening-up-the-nhs/.

10 The Mid Staffordshire NHS Foundation Trust Public Inquiry. 2013. www. midstaffspublicinquiry.com.

Cite this as: BMJ 2013;346:f1601

๑ BMJ Publishing Group Ltd 2013 


\section{Table}

Table 1| UK antimicrobial resistance strategy: seven action areas and likely stakeholder involvement in the health sector

Seven key areas of focus

Promote responsible evidence based prescribing

mprove infection prevention and control

Raise public and professional awareness of antimicrobial resistance threat and promote behaviour change

Research programme into new diagnostics, alternatives to antibiotics (such as antiseptics), pathogenesis, effective behavioural change to improve infection prevention and control and prescribing practice

Facilitate development of new antimicrobials, vaccines, and immunomodulators

Improve surveillance and data linkage

Encourage international collaboration and data sharing and learning from best practice Department of Health, PHE

internationally

\section{Stakeholders}

Individual prescribers, NHS providers, national and local commissioning boards, ARHAI, PHE, Department of Health, professional bodies

Individual clinical staff, NHS providers, national and local commissioning boards, ARHAI, Department of Health, PHE, professional bodies

Professional bodies, Department of Health, ARHAI, patient groups

NIHR, universities, Department of Health, ARHAI

Department of Health, drug industry, European Union

PHE, ARHAI, Department of Health

ARHAl=Department of Health Expert Advisory Committee on Antimicrobial Resistance and Healthcare Associated Infection; PHE=Public Health England. 